\title{
Cloning and expression analysis of PpSUT2 encoding a sucrose transporter in pear
}

\author{
J. Tang', ${ }^{1,2}$ J. Lin ${ }^{1}$, B.L. Zhang ${ }^{1}$, Z.H. Wang', X.G. Li ${ }^{1}$ and Y.H. Chang ${ }^{1}$ \\ ${ }^{1}$ Institute of Horticulture, Jiangsu Academy of Agricultural Sciences, \\ Nanjing, China \\ ${ }^{2}$ College of Horticulture, Nanjing Agricultural University, Nanjing, China \\ Corresponding authors: Y.H. Chang / J. Lin \\ E-mail: cyh@jaas.ac.cn / 1j84390224@126.com
}

Genet. Mol. Res. 13 (4): 8932-8945 (2014)

Received August 8, 2013

Accepted December 12, 2013

Published October 31, 2014

DOI http://dx.doi.org/10.4238/2014.October.31.8

\begin{abstract}
A 1794-bp cDNA fragment was amplified from mRNA isolated from pear (Pyrus pyrifolia NaKai. Cuiguan) leaves by using primers based on the sequences generated during the analysis of the pear transcriptome. The 597-amino acid sequence encoded by the cDNA was compared with the sequences in GenBank, and it was found to be similar to that of members of the sucrose-proton cotransporter family. The hydrophobic protein, which was predicted to have 11 transmembrane domains, was designated as PpSUT2. Realtime fluorescent quantitative polymerase chain reaction analysis indicated the accumulation of PpSUT2 mRNA throughout the plant, with the highest levels in the buds. Analysis of the expression of PpSUT2 during fruit development showed that the abundance of its transcripts increased at the end of April and then decreased to the lowest level at the end of July. Subcellular localization studies with the pCXDG vector as a probe demonstrated that PpSUT2 localized to cell membranes. An expression vector was constructed by inserting the PpSUT2 cDNA into pET32(a), and the vector was expressed in Escherichia coli (strain BL21) after induction with $1 \mathrm{mM}$ isopropyl $\beta$-D-1-thiogalactopyranoside at $25^{\circ} \mathrm{C}$. Analysis using sodium dodecyl
\end{abstract}


sulfate-polyacrylamide gel electrophoresis identified the induction of a 71-kDa protein. Further analysis indicated that PpSUT2 might be not directly involved in sucrose transport, instead, functioning as a sucrose sensor on the cytoplasmic membrane.

Key words: Sucrose transporter; PpSUT2; Subcellular localization; Real-time fluorescent quantitative polymerase chain reaction; Prokaryotic expression

\section{INTRODUCTION}

Sugars, which are synthesized during photosynthesis, are essential for plant growth and development. The type and composition of sugars in fruit have a considerable impact on their sweetness and flavor (Tao et al., 2010). The three major soluble sugars in pear fruit are sucrose, glucose, and fructose (Forney and Breen, 1985). Sucrose is one of the major substances transported from the sources to sinks in photosynthesis (Yamaki, 1995). The sucrose transporter SUT imports sucrose from source tissues into fruit (Yamaki, 1995). The manner of sucrose transportation and its distribution not only help to coordinate growth and development through regulating intermediary metabolism and gene expression but also determine the yield and quality of the economically important parts of the crop. Sucrose transporters play a key role in determining the direction of source-to-sink transport.

Phylogenetic analysis of sucrose transporters demonstrated that they could be classified into the three subgroups: SUT1, SUT4, and SUT2 (Kühn, 2003). In higher plants, members of the SUT2 subgroup are highly diversified in their biological functions. The similarities of protein sequences of sucrose transporters were higher among dicotyledonous plants than among monocotyledonous plants (Yang et al., 2006). Among all of the SUT2 isoforms that were identified in monocots, only bSUT2 has special cytoplast domains that are similar to those found in SUT2 isoforms from dicots. Furthermore, no sucrose transportation activity has been found for dicot StSUT2 and LeSUT2, whereas AtSUT2 has been shown to have the lowest affinity for sucrose (Barker et al., 2000; Schulze et al., 2000; Aoki et al., 2003). It is speculated that SUT2 is a sucrose sensor on the cytoplasmic membrane (Barker et al., 2000), which can use sucrose signaling to regulate the expression of the SUT4 gene and as the folding and activity of the SUT4 protein (Barker et al., 2000; Weise et al., 2000).

Several reports have described sucrose transporters (Hackel et al., 2006; Peng, 2006), but the isolation and characterization of SUT2 from pear has not been reported. PpSUT2 is an important gene in the plant sucrose synthesis pathway. In this study, we isolated and characterized pear SUT2 and its biological function in an attempt to understand more about the mechanisms that control sucrose accumulation in this commercially important species.

\section{MATERIAL AND METHODS}

\section{Materials}

Tissue samples were taken from various parts of pear (Pyrus pyrifolia NaKai. Cuiguan) trees, including the flower, young leaf, stem, bud, and fruit. The pear trees were grown in a germ- 
plasm nursery at the Horticulture Institute at Jiangsu Agricultural Academy, Nanjing. Samples of flower, young leaf, stem, and bud were collected on March 25, 2011, and the first batch of fruit samples was collected on April 10, 2011, with subsequent samples collected at 10-day intervals. The samples were frozen in liquid nitrogen and stored at $-70^{\circ} \mathrm{C}$ until subsequent use.

\section{Cloning of PpSUT2}

Total RNA was extracted from the collected tissue samples using a cetyltrimethylammonium bromide method (Luo et al., 2001). Primers were designed based on the sequences available from pear transcriptome analysis to amplify cDNA from the leaf tissue. An amplicon of the expected size was cloned for analysis. The polymerase chain reaction (PCR) product was recovered, purified, and ligated into the pGEM-T easy vector (TaKaRa, Dalian, China). The ligated product was used to transform Escherichia coli DH5a competent cells, which were grown on Luria-Bertani (LB) plates containing $100 \mathrm{mg} / \mathrm{mL}$ ampicillin to select colonies for sequence analysis.

\section{Sequence analysis}

DNA sequences obtained from sequencing some selected colonies were analyzed using DNAclub for the presence of open reading frames and were aligned for sequence similarity using BioEdit (an applied Molecular Biology Software). The sequences were also searched against the DNA sequences in public GenBank using the basic local alignment search tool (BLAST) algorithm (http://blast.ncbi.nlm.nih.gov/Blast.cgi) to identify homologs (Yang et al., 2011). Using DNAMAN to analyze amino acid homology, a phylogenetic tree was constructed to represent the relatedness of known SUT2 sequences using Mega 5.0 (Tamura et al., 2011). The molecular weight and theoretical isoelectric point (pI) of the protein were predicted using ProtParam (http://web.expasy.org/protparam/), its transmembrane domains were predicted using TMHMM (http://www.cbs.dtu.dk/services/TMHMM/), and its subcellular localization was predicted using PSORT (http://psort.hgc.jp/form.html).

\section{Fluorescent quantitative reverse transcription (RT)-PCR}

RNA from various parts of the plant was treated with RQ1 DNase (Promega, Beijing, China) and reverse transcribed into cDNA using oligo(dT) primers. RT-PCR was carried out using the SYBR Premix EX Taq kit purchased from TaKaRa according to manufacturer instructions. Internal references were amplified using the primers based on the pear $\beta$-actin gene with the following sequences: P1r: 5'-GAATGGTCAAGGCTGGGTT-3' and P1f: 5'-CAAAGCATCTGAGGTCA-3' (Li et al., 2010). RT-PCR was used to quantify the expression of PpSUT2. The following thermal cycling conditions were used for PCR; $95^{\circ} \mathrm{C}$ for 1 min, followed by 40 cycles of $95^{\circ} \mathrm{C}$ for $30 \mathrm{~s}, 58^{\circ} \mathrm{C}$ for $20 \mathrm{~s}$, and $72^{\circ} \mathrm{C}$ for $20 \mathrm{~s}$.

\section{Subcellular localization}

\section{Materials, reagents, and instruments}

E. coli strain DH5 $\alpha$ was obtained from the Institute of Biotechnology, Jiangsu Agri- 
cultural Academy, Nanjing. T4 DNA ligase and high fidelity DNA polymerase were purchased from TaKaRa, the pCXDG vector was purchased from HQ-biotech (Beijing, China), the DNA gel recovery kit was purchased from Shanghai Bo Biotechnology, and reverse transcriptase and RNA inhibitors were purchased from Promega.

\section{Construction of pCXDG-PpSUT2 for transient expression}

Primers were designed based on the sequences of pCXDG (Chen et al., 2009) and PpSUT2 to amplify the coding region using plasmid DNA containing the gene as template. The amplified products were recovered from the gel and ligated into the pCXDG expression vector. The ligation reaction was transformed into DH5 $\alpha$ competent cells, and transformants were selected on LB plates containing $50 \mu \mathrm{g} / \mathrm{mL}$ kanamycin (Sambrook and Russell, 2001). The orientations of the ligation were verified by PCR amplification, and the identities of positive clones were confirmed by sequencing.

\section{Agrobacterium-mediated transformation and subcellular localization}

The pCXDG-PpSUT2 recombinant vector was transformed into Agrobacterium cells (strain EHA4404) using the freeze-thaw method and selected on LB plates containing $50 \mu \mathrm{g} / \mathrm{mL}$ rifamycin and $50 \mu \mathrm{g} / \mathrm{mL}$ kanamycin (Eady et al., 2005). The transformed colonies were further confirmed by PCR and grown in LB liquid medium containing $50 \mu \mathrm{g} / \mathrm{mL}$ rifamycin and $50 \mu \mathrm{g} / \mathrm{mL}$ kanamycin for $36 \mathrm{~h}$. The primer sequences were P2r: 5'-TTACCCAAAATGAAAACCCGTTG-3' and P2f: 5'-GATGGCGGGGAGGACGGACT-3'. The bacterial cells were pelleted by spinning at $4000 \mathrm{rpm}$ for $8 \mathrm{~min}$ and were re suspended in Agrobacterium inoculation solution (LB medium with $10 \mathrm{mM} \mathrm{MgCl}_{2}$ and $100 \mu \mathrm{M}$ acetosyringone) to an optical density at a wavelength of $600 \mathrm{~nm}$ (OD600) of 0.6. Onion inner epidermal tissues were dipped into the Agrobacterium inoculation solution for 20-30 min, blotted dry on sterile filter paper, and cultured on solid Murashige and Skoog medium at $26^{\circ} \mathrm{C}$ for $16 \mathrm{~h}$. The co-cultivated tissues were washed with sterile water and placed on slides for observation using an inverted microscope (IX71-F22PH).

\section{Construction of the prokaryotic expression vector for PpSUT2 and its induced expression}

\section{Construction of the prokaryotic expression vector}

The PpSUT2 prokaryotic expression vector was constructed, and its expression products were analyzed using the following the procedure that was described previously (Tameling et al., 2002). The primers used were P3r: 5'-ACAGAGCTCATGGCGGGGAGGACGGACT-3' and P3f: 5'-ACACTCGAGTTACCCAAAATGAAAACCCGTT-3'. The following conditions for thermal cycling were used: $94^{\circ} \mathrm{C}$ for $3 \mathrm{~min} ; 35$ cycles of $94^{\circ} \mathrm{C}$ for $30 \mathrm{~s}, 57^{\circ} \mathrm{C}$ for $40 \mathrm{~s}$, and $72^{\circ} \mathrm{C}$ for $2 \mathrm{~min}$; and $72^{\circ} \mathrm{C}$ for $10 \mathrm{~min}$. The amplified products and the pET32(a) plasmid DNA were double-digested using the restriction enzymes XhoI and SacI, and they were gelpurified. The recovered DNA fragments were ligated using T4 DNA ligase and transformed into DH5 $\alpha$ cells. Plasmid DNA was prepared from the resistant colonies to identify the correct 
recombinant vector, which was then used to transform cells of the E. coli BL21 expression strain.

\section{Isopropyl $\beta$-D-1-thiogalactopyranoside (IPTG)-induced expression}

A single colony was inoculated into LB medium containing $100 \mu \mathrm{g} / \mathrm{mL}$ ampicillin and grown to OD600 $=0.6$ at $37^{\circ} \mathrm{C}$ and $200 \mathrm{rpm}$ on a shaker. IPTG was added to a final concentration of $1 \mathrm{mM}$, and growth was allowed to proceed at $25^{\circ} \mathrm{C}$ on a shaker operated at $180 \mathrm{rpm}$. A cell line that carried empty pET32(a) without PpSUT2 was a negative control. Aliquots of the culture were taken $0,12,24$, and $36 \mathrm{~h}$ after the addition of IPTG (induction), and bacterial cells in the aliquots were precipitated by centrifugation, resuspended in phosphate-buffered saline solution, and ruptured by sonication. The protein extracts were resolved by electrophoresis using $8 \%$ sodium dodecyl sulfate-polyacrylamide gel electrophoresis (SDS-PAGE) as previously described (Baneyx, 1999; Han et al., 2002).

\section{RESULTS}

\section{Molecular cloning and analysis of PpSUT2}

To amplify the coding sequence of the SUT2 gene from pear, DNA sequences in the pear transcriptome were searched to identify the sequences that encoded homologs of known SUT2 sucrose transporters, and then these sequences were used to design primers to amplify the pear SUT2 cDNA (Figure 1). The amplified product was ligated to the pGEM-T easy vector, and sequenced. The full-length cDNA (1794 bp) encoded a 597-amino acid protein that was predicted to have 11 transmembrane domains (Figure 2).

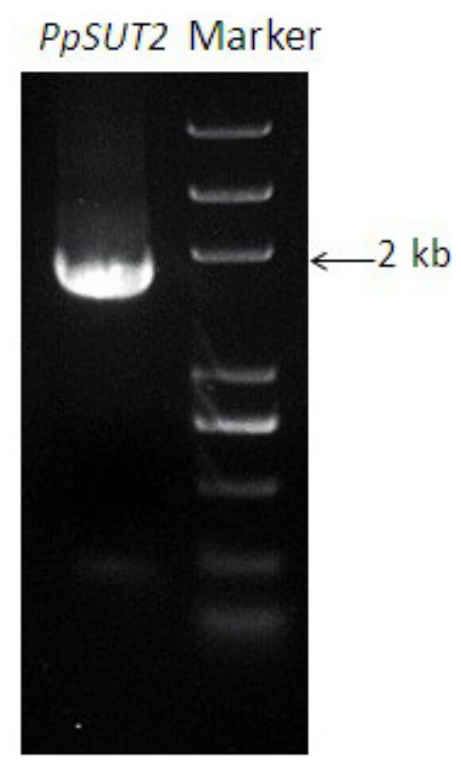

Figure 1. Polymerase chain reaction amplification product of PpSUT2. 


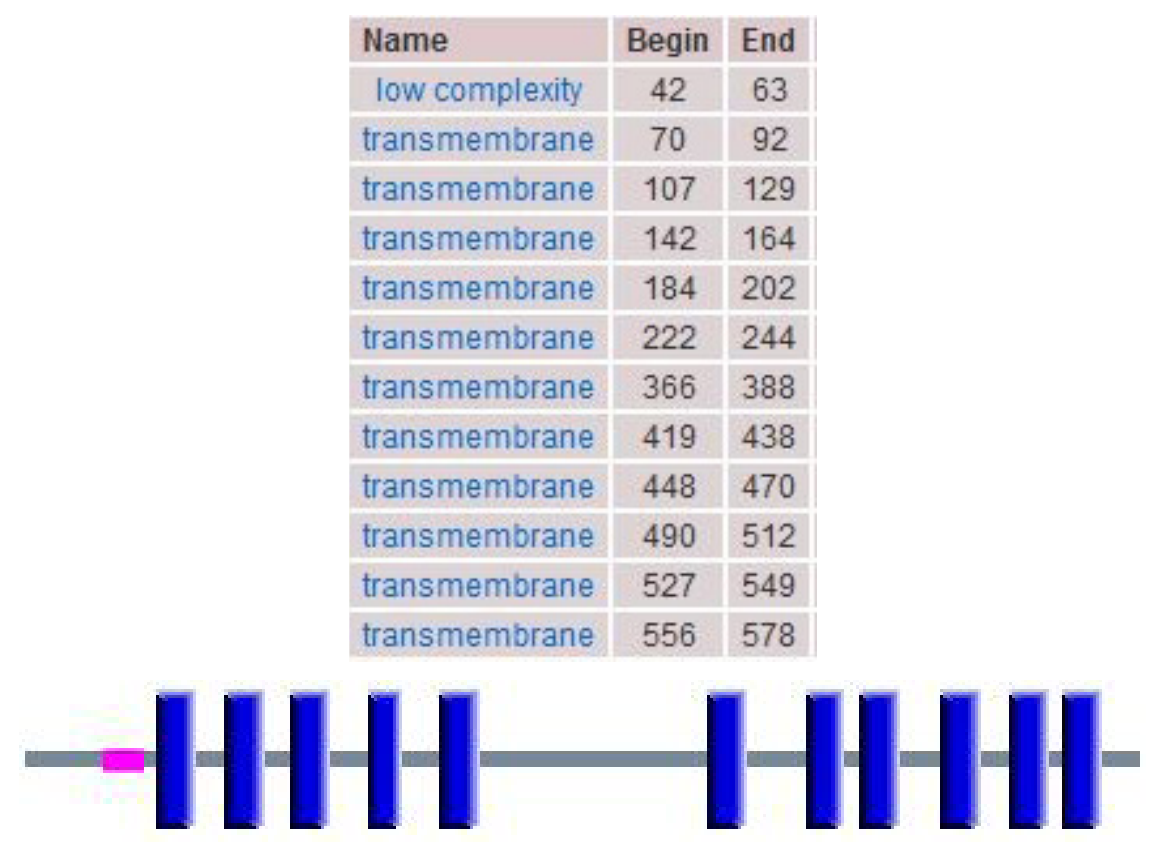

Figure 2. Predicted transmembrane domains in PpSUT2 and their start and end positions.

Use of the BLAST algorithm to compare the deduced amino acid sequence of the clone with sequences in GenBank revealed that the gene belongs to the GPH-sucrose proton co-transporter superfamily (Figure 3). The gene was therefore designated as PpSUT2. Cluster analysis of PpSUT2 with SUT2 sequences of the six above-mentioned plants indicated that PpSUT2 showed the greatest similarity to the SUT2 sequences from strawberry, grape, and orange (1. Medicago truncatula; 2. Zea mays; 3. Fragaria x ananassa; 4. Zea mays(2); 5. Manihot esculenta; 6. Hordeum vulgare subsp vulgare). The amino acid sequence of the PpSUT2 transmembrane region was highly conserved among different species (Figure 4). The phylogenetic tree analysis indicated that PpSUT2 was in the same family as maize SUT2, which is the second group in the SUT family with a highly similar function (Figure 5).

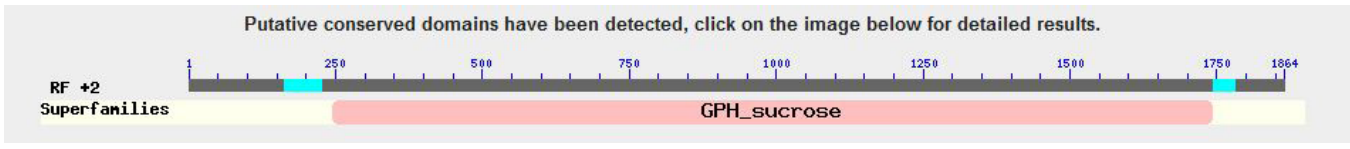

Figure 3. Basic local alignment search tool results for conserved domains in PpSUT.

\section{Prediction and analysis of PpSUT2 transmembrane domains}

Given that transmembrane domains are the sites of protein and membrane lipid in- 
teractions, their prediction and analysis may provide important clues regarding the structures and functions of many plant proteins. Using the TMHMM Server v.2.0, we detected 11 transmembrane domains in PpSUT2, which was consistent with its classification as a member of the proton co-transporter superfamily (Figure 6). For such analyses, each amino acid residue in a protein was given a value to measure its hydrophobicity, with a value between 0 and 4.5 denoting hydrophobicity and a value between 0 and -4.5 denoting hydrophilicity. PpSUT2 has values between -2.5 and 2.5. The hydrophobicity of PpSUT2 indicates that it contains a high proportion of hydrophobic amino acids, a requirement for transmembrane domain formation (Figure 7).
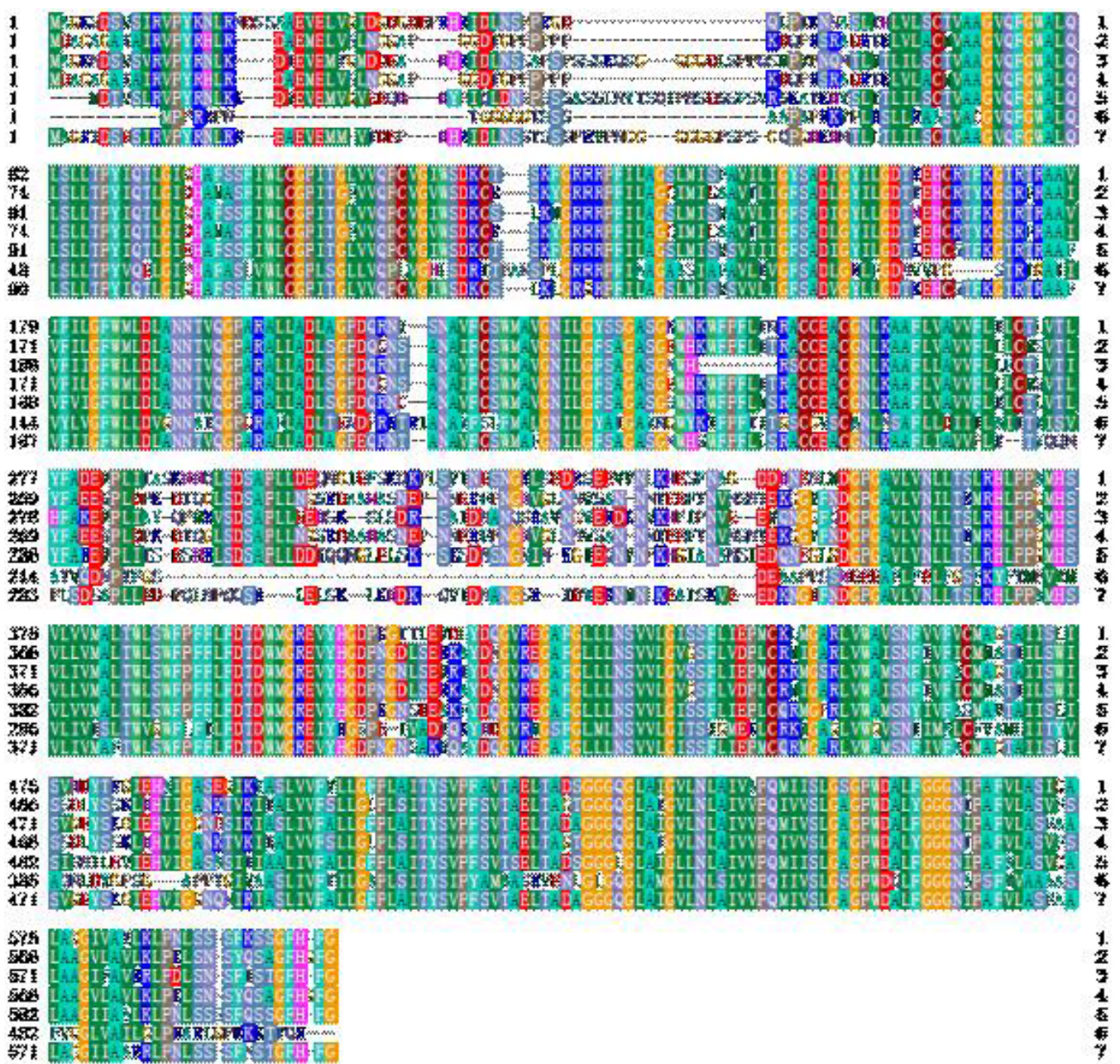

Figure 4. Alignment of the deduced amino sequence of PpSUT2 with those from other species. 1. Medicago truncatula; 2. Zea mays; 3. Fragaria $\mathrm{x}$ ananassa; 4. Zea mays(2); 5. Manihot esculenta; 6. Hordeum vulgare subsp vulgare; and 7. Pyrus pyrifolia NaKai. Cuiguan. 


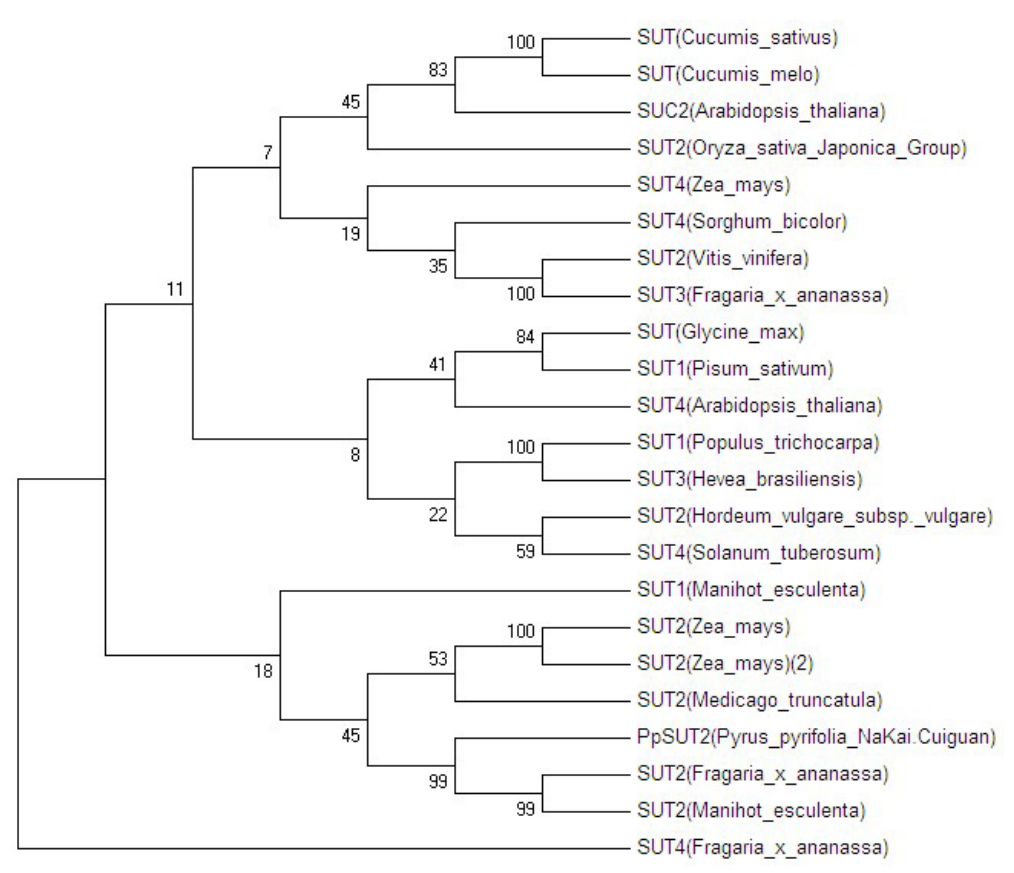

Figure 5. Phylogenetic analysis of PpSUT2 and other SUT2 isoforms from plants. The numbers indicate the bootstrap values from 1000 replicates. The bar represents 5\% sequence divergence. The tree is based on maximum parsimony analysis for protein sequences of aligned sucrose transporters from Arabidopsis thaliana (AEE30276.1, AAG09192.1); Populus trichocarpa (ADW94613.1); Manihot esculenta (ABA08445.1,ABA08446.1); Glycine max (CAD91334.1); Pisum sativum (AAD41024.1); Medicago truncatula (AFM28287.1); Zea mays (NP 001137486.1, AAS91375.1, NP_001146651. 1); Fragaria $\mathrm{x}$ ananassa (AFU61910.1, AFU61909.1, AFU61908.1); Oryza sativa Japonica Group (ADZ23999.1); Vitis vinifera (XP_002266122.1); Hordeum vulgare subsp vulgare (CAB75881.1); Hevea brasiliensis (ABK60190.2); Solanum tuberosum (AAG25923.2); Cucumis sativus (ADB04246.1); Cucumis melo (ACJ04700.1); Sorghum bicolor (ACX71839.1); and PpSUT2 (Pyrus pyrifolia NaKai.Cuiguan).

TMHMM posterior probabililties for sequence

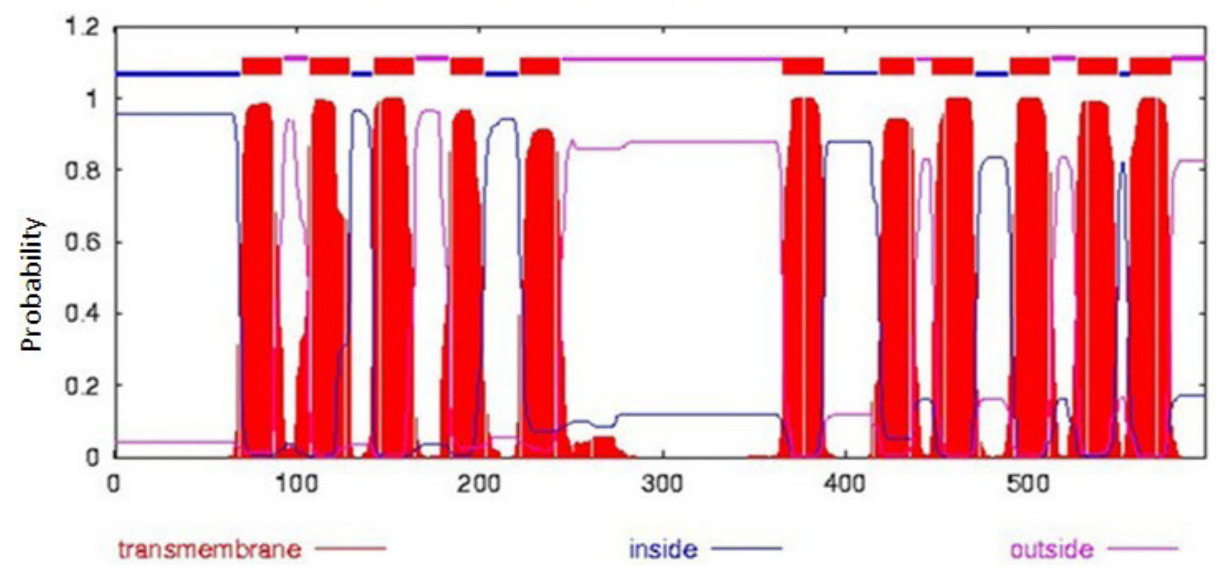

Figure 6. Topology prediction of PpSUT2. 


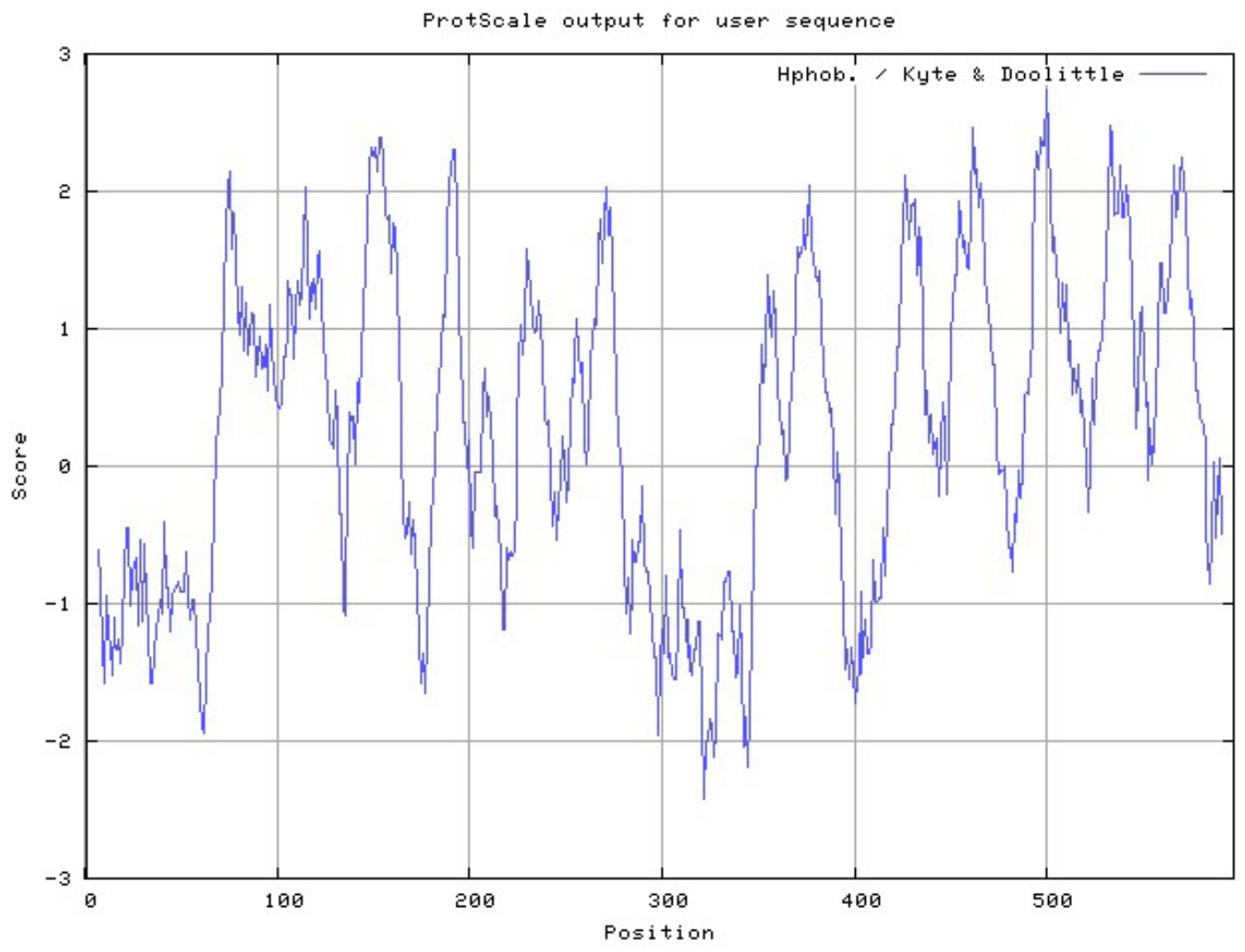

Figure 7. Hydrophobicity plot calculated from the amino acid sequence of the sucrose transporter that was deduced from the nucleotide sequence of PpSUT2.

\section{Expression profiling of PpSUT2}

The investigation of PpSUT2 expression revealed the accumulation of PpSUT2 transcripts in all pear tissues that were studied, albeit with considerable variation in spatial and temporal patterns of expression. Higher levels were detected in young leaves, buds, and immature fruits. The level of PpSUT2 transcripts in young leaves was about six times higher than in stems (Figure 8). During fruit development, PpSUT2 expression levels increased during the early development stage of fruit (10 days after flowering), reached a maximal level 20 days after flowering, and then decreased rapidly to a low level that was maintained until maturity (Figure 9).

\section{Characterization of the subcellular location of PpSUT2 using the transient expression vector $\mathrm{pCXDG}-P$ PSUT2}

The prediction of subcellular localization by PSORT showed that the probabilities of its localization on the cytoplasmic membrane, chloroplastic thylakoid membrane, and 
mitochondrial inner membrane were $80.0,53.2$, and $37.2 \%$, respectively. To further study the cellular location of PpSUT2, the coding sequence was fused in frame to the coding sequence for GFP in the vector pCXDG. Colonies containing putative fusion genes were grown overnight on LB plates containing kanamycin, and resistant colonies were analyzed by PCR. One of the colonies selected could produce a fragment with the expected size of $2.0 \mathrm{~kb}$ (Figure 10).

The recombinant vector pCXDG-PpSUT2 was then transformed into onion epidermal tissue using Agrobacterium-mediated transformation. After overnight co-cultivation, the tissue was observed at $480 \mathrm{~nm}$ using a fluorescent microscope. GFP signals were clearly visible on onion cell membranes, but not in other parts of the cells, indicating that PpSUT2 was targeted to the cell membranes of onion cells (Figure 11). This experimental evidence is consistent with the location that was predicted using PSORT.

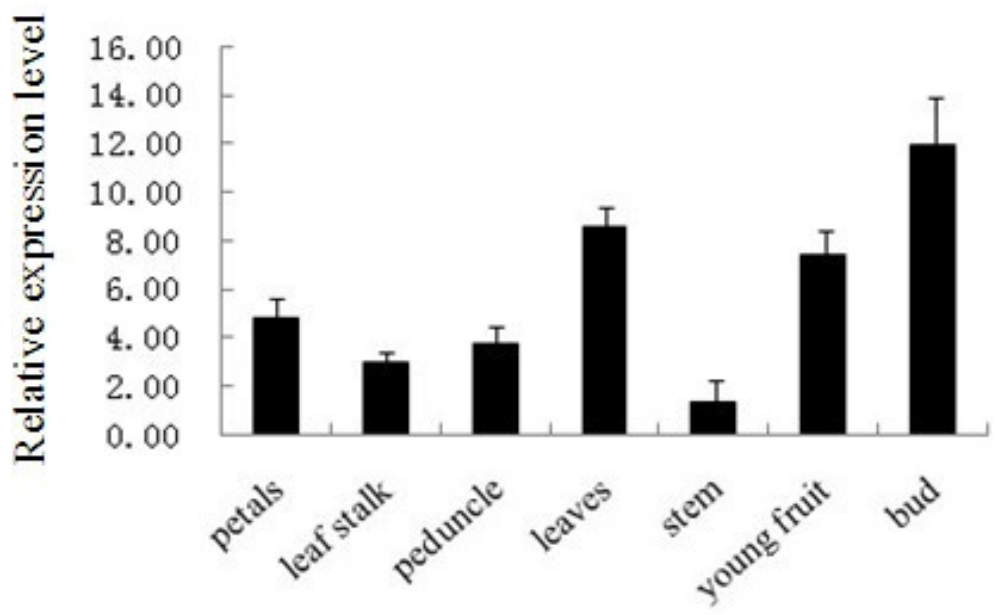

Figure 8. Relative expression of the PpSUT2 gene in different pear tissues.

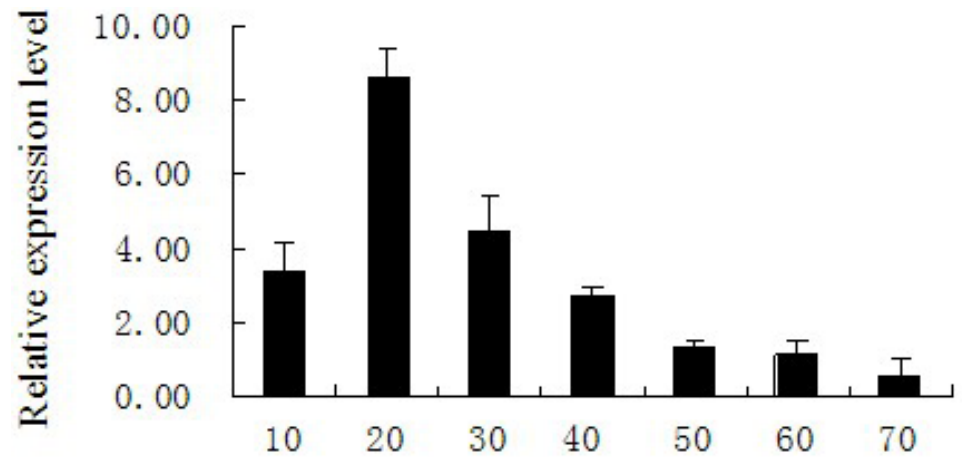

\section{Days after full bloom (d)}

Figure 9. Relative expression of the PpSUT2 gene in pear fruit at different times after flowering. 


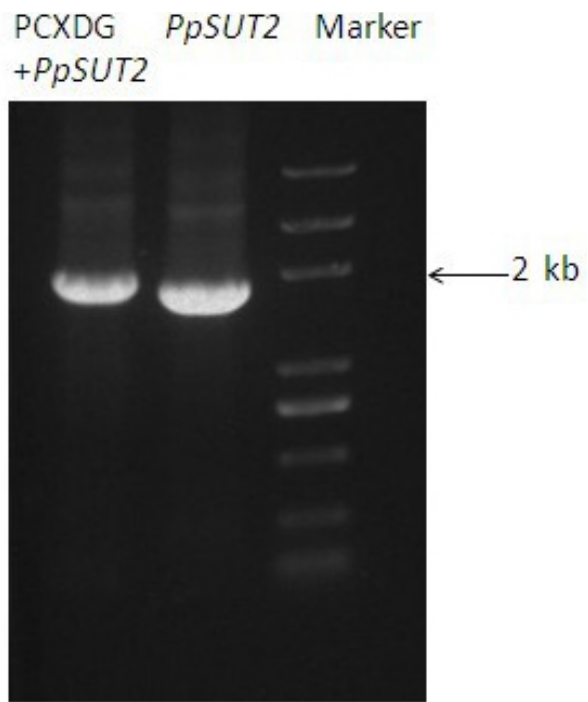

Figure 10. Analysis of the pCXDG-PpSUT2 recombinant vector by agarose gel electrophoresis.

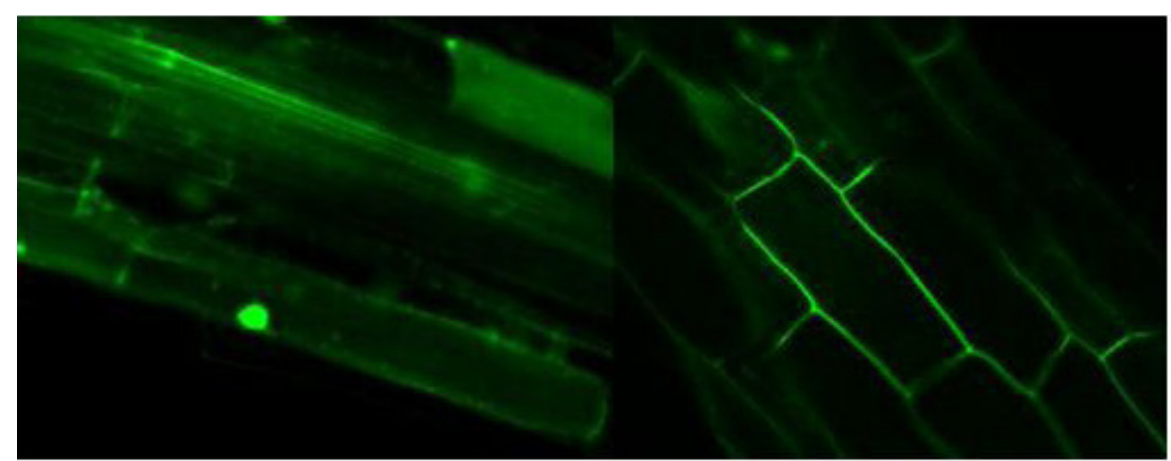

A

B

Figure 11. Transient expression of the GFP-PpSUT2 fusion vector in onion epidermal cells after Agrobacteriummediated transformation. A. GFP fluorescence from pCXDG. B. GFP fluorescence from the GPF-PpSUT2 fusion vector.

\section{Prokaryotic expression of PpSUT2}

The complete coding sequence of PpSUT2 was amplified from pear (P. pyrifolia NaKai. Cuiguan) cDNA using the primers P3r and P3f. After digestion with XhoI and SacI, the coding region was ligated to the $E$. coli expression vector pET32(a) that had been digested with the same two restriction enzymes. The resultant plasmid was analyzed by restriction enzyme mapping (Figure 12). The expression vector was transformed into E. coli (BL21) cells along with empty vector, expression was induced by adding $1 \mathrm{mM} \mathrm{IPTG}$, and the cells were grown in a shaker at $25^{\circ} \mathrm{C}$. Aliquots of the culture were taken at different times after the induction. It was speculated that the 
protein might be a membrane protein and would accumulate as insoluble inclusion bodies in $E$. coli cells. As such, the cells were ruptured by sonication before being extracted for SDS-PAGE. The results indicated that there was a slight increase in the abundance of a protein of the predicted size $(70 \mathrm{kDa}) 12 \mathrm{~h}$ after induction with $1 \mathrm{mM}$ IPTG, and the band intensity continued afterward (Figure 13). No such band was seen after electrophoresis of protein extracts from cells containing empty vector or cells containing the recombinant vector that had not been exposed to IPTG.

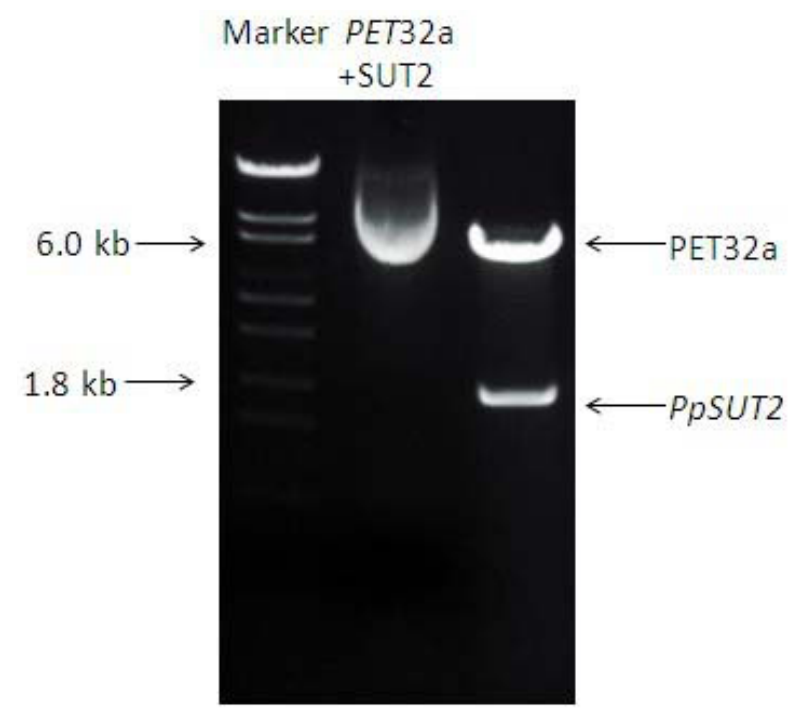

Figure 12. Enzyme digestion verification of the prokaryotic PpSUT2 expression vector.

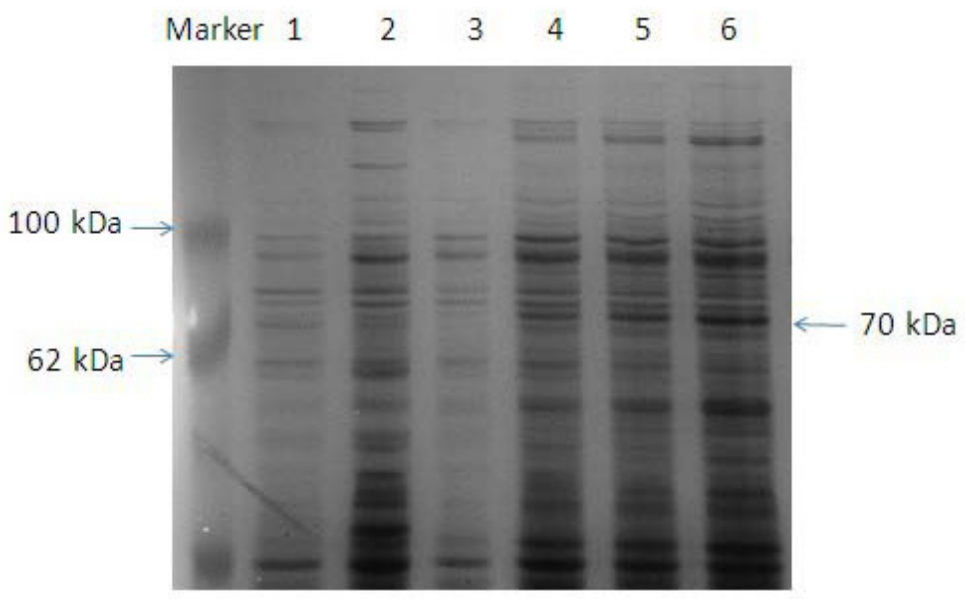

Figure 13. Sodium dodecyl sulfate-polyacrylamide gel electrophoresis analysis of the expression of PpSUT2 in the pET32(a) vector. Lane Marker = protein molecular weight markers; lane 1 = proteins extracted from uninduced pET32(a) empty vector; lane 2 = proteins extracted from pET32(a) empty vector after induction with $1 \mathrm{mM}$ isopropyl $\beta$-D-1-thiogalactopyranoside (IPTG) at $25^{\circ} \mathrm{C}$ for $36 \mathrm{~h}$. Lanes $3-6=$ proteins extracted from pET32(a)PpSUT2 after induction with $1 \mathrm{mM} \mathrm{IPTG}$ at $25^{\circ} \mathrm{C}$ for $0,12,24$, and $36 \mathrm{~h}$, respectively. 


\section{DISCUSSION}

Plant SUT2 proteins are believed to function as signal proteins that control the transport of sucrose across membranes (Barker et al., 2000; Schulze et al., 2000; Reinders et al., 2005) by sensing sucrose signals that directly regulate the expression, folding, and activities of SUT1 and SUT4. This regulates the sucrose content of plant fruit (Barker et al., 2000; Weise et al., 2000). Given the importance of cloning SUT2 genes to better understand the regulation of sucrose synthesis, this study involved the isolation of a 1794-bp SUT2 cDNA from the pear variety Green Crown using primers based on the analysis of the pear transcriptome. Amino acid sequence analysis indicated that PpSUT2 belongs to a superfamily of sucrose-proton co-transporters.

In comparison with SUT2 sequences from a few species that, evolutionarily, are closely related to pear, PpSUT2 showed high similarity to SUT2 proteins from strawberry, grape, and Zea mays and that it has less similarity to SUT2 from cucumber. This is consistent with the phylogenetic relationships among these plants. Phylogenetic analysis indicated that PpSUT2 and corn SUT2 clustered in the same SUT subfamily (Barker et al., 2000; Weise et al., 2000), which suggests that they serve very similar functions in these species.

Analysis using qRT-PCR indicated that the PpSUT2 gene was expressed in various tissues at different levels; the highest levels of PpSUT2 transcripts were found in young leaves, and the lowest levels were found in stems. Expression in buds was six times higher than that in stems. Higher levels of PPSUT2 transcripts in young tissues and lower levels in mature tissue are consistent with previous reports of the differential expression of genes that encode plant sucrose transporters (Hackel et al., 2006). In pear fruit, it was found that expression of PpSUT2 increased at 20 days after full blooms, and then the expression reduced gradually. Seventy days after full blooms, the expression was at its lowest level. This seems to be inversely related to the sugar content in pear fruit, with increased sugar content reducing the levels of gene expression. Studies indicated that decreases in the expression of PpSUT2 accompany the ripening of fruit and the accumulation of sugar. Meanwhile, pear fruit is known to contain higher amounts of sucrose at later developmental stages than early during fruit development (Hackel et al., 2006). Therefore, we speculate that the PpSUT2 gene is not directly involved in sucrose transportation, and it is more likely to act as a sensor of a sucrose-related signal.

To study the cellular location of PpSUT2, we constructed a GFP-PpSUT2 fusion protein expression vector. After Agrobacterium-mediated transformation of onion cells with this construct, we observed the localization of GFP on the cell membrane, indicating that the protein might be a membrane-associated protein. This is consistent with bioinformatic predictions. Our analysis indicated that P PSUT2 encodes a membrane-bound protein and might play an important role in sucrose accumulation.

After optimizing the induction time and temperature, a protein of the expected molecular size $(70 \mathrm{kDa})$ was isolated from $E$. coli BL21 cells carrying the fusion protein construct, but it was not isolated from the empty vector or without induction. This implies that the protein is the expected fusion protein. Amino acid sequence analysis showed that the protein has 11 transmembrane domains, indicating that the protein is a membrane protein, which typically exists in inclusion bodies when expressed in E. coli cells. A high content of hydrophobic amino acids in a hydrophobic protein is very conducive to the formation of transmembrane domains. The presence of the expressed PpSUT2 protein in inclusion bodies will likely complicate its purification. Nonetheless, the successful purification of the protein will facilitate 
its further characterization and permit the preparation of an antibody for use in immunohistochemical analysis, protein blot analysis, and immunoprecipitation experiments.

\section{ACKNOWLEDGMENTS}

We are grateful for the help that Professor Fang Jing-gui provided throughout the article writing process. Research supported by grants from the Natural Science Foundation of Jiangsu Province (\#BK2010472 and \#BK2011674).

\section{REFERENCES}

Aoki N, Hirose T, Scofield GN, Whitfeld PR, et al. (2003). The sucrose transporter gene family in rice. Plant Cell Physiol. 44: 223-232.

Baneyx F (1999). Recombinant protein expression in Escherichia coli. Curr. Opin. Biotechnol. 10: 411-421.

Barker L, Kuhn C, Weise A, Schulz A, et al. (2000). SUT2, a putative sucrose sensor in sieve elements. Plant Cell 12: 1153-1164.

Chen S, Songkumarn P, Liu J and Wang GL (2009). A versatile zero background T-vector system for gene cloning and functional genomics. Plant Physiol. 150: 1111-1121.

Eady C, Davis S, Catanach A, Kenel F, et al. (2005). Agrobacterium tumefaciens-mediated transformation of leek (Allium porrum) and garlic (Allium sativum). Plant Cell Rep. 24: 209-215.

Forney CF and Breen PJ (1985). Collection and characterization of phloem exudate from strawberry pedicels. Hortscience 20: 413-414.

Hackel A, Schauer N, Carrari F, Fernie AR, et al. (2006). Sucrose transporter LeSUT1 and LeSUT2 inhibition affects tomato fruit development in different ways. Plant J. 45: 180-192.

Han CG, Li DW, Yu JL, Liu L, et al. (2002). Preparation and application of specific antiserum against wheat yellow mosaic virus coat protein expressed in E. coli cells. J. Agric. Biotechnol. 10: 373-376.

Kühn C (2003). A comparison of the sucrose transporter systems of different plant species. Plant Biol. 5: 215-232.

Li H, Cong Y, Wang HW, Sheng B, et al. (2010). Molecular cloning and expression analysis of a phytochelatin synthase gene, PcPCS1, from Pyrus calleryana Dcne. Acta Hortic. Sin. 37: 880-890.

Luo P, Wang GD and Chen XY (2001). Isolation and expression analysis of two cDNAs encoding C4H homologues from Gossypium arboreum. Acta Bot. Sin. 43: 77-81.

Peng CC (2006). Gene Cloning and Interacting Partner Screening of Sucrose Transporter in Apple Fruit. China Agricultural University, Beijing.

Reinders A, Panshyshyn JA and Ward JM (2005). Analysis of transport activity of Arabidopsis sugar alcohol permease homolog AtPLT5. J. Biol. Chem. 280: 1594-1602.

Sambrook J and Russell DW (2001). The Condensed Protocols from Molecular Cloning: A Laboratory Manual. Cold Spring Harbor, New York.

Schulze W, Weise A, Frommer WB and Ward JM (2000). Function of the cytosolic N-terminus of sucrose transporter AtSUT2 in substrate affinity. FEBS Lett. 485: 189-194.

Tameling WI, Elzinga SD, Darmin PS, Vossen JH, et al. (2002). The tomato R gene products I-2 and MI-1 are functional ATP binding proteins with ATPase activity. Plant Cell 14: 2929-2939.

Tamura K, Peterson D, Peterson N, Stecher G, et al. (2011). MEGA5: molecular evolutionary genetics analysis using maximum likelihood, evolutionary distance, and maximum parsimony methods. Mol. Biol. Evol. 28: 2731-2739.

Tao H, Cui JF and Nie LC (2010). Research process on sugar accumulation in fruit. J. Anhui Agri. Sci. 38: 42-44.

Weise A, Barker L, Kuhn C, Lalonde S, et al. (2000). A new subfamily of sucrose transporters, SUT4, with low affinity/ high capacity localized in enucleate sieve elements of plants. Plant Cell 12: 1345-1355.

Yamaki S (1995). Physiology and metabolism of fruit development - biochemistry of sugar metabolism and compartmentation in fruits. Acta Hort. 398: 109-120.

Yang CJ, Hao DHi and Yang SX (2006). Sucrose transporters in higher plants. Plant Physiol. J. 42: 767-776.

Yang YW, He B, Zhang BL and Ni WC (2011). Molecular cloning and expression analysis of a receptor-like protein kinase gene in upland cotton. Cotton Sci. 23: 15-21. 